\title{
RECAPTURED PHOTO DETECTION USING SPECULARITY DISTRIBUTION
}

\author{
Hang Yu, Tian-Tsong Ng and Qibin Sun \\ Institute for Infocomm Research $\left(I^{2} R\right), \mathrm{A} *$ STAR, Singapore, 119613 \\ \{hyu, ttng, qibin\}@i2r.a-star.edu.sg
}

\begin{abstract}
Detection of planar surfaces in a generic scene is difficult when the illumination is complex and less intense, and the surfaces have non-uniform colors (e.g., a movie poster). As a result, the specularity, if appears, is superimposed with the surface color pattern, and hence the observation of uniform specularity is no longer sufficient for identifying planar surfaces in a generic scene as it does under a distant point light source. In this paper, we address the problem of planar surface recognition in a single generic-scene image. In particular, we study the problem of recaptured photo recognition as an application in image forensics. We discover that the specularity of a recaptured photo is modulated by the microstructure of the photo surface, and its spatial distribution can be used for differentiating recaptured photos from the original photos. We validate our findings in real images of generic scenes. Experimental results show that there is a distinguishable feature of natural scene and recaptured images. Given the definition of specular ratio as the percentage of specularity in the overall measured intensity, the distribution of specular ratio image's gradient of natural images is Laplacian-like while that of recaptured images is Rayleigh-like.
\end{abstract}

Index Terms - Image forensics, recaptured photo detection, dichromatic reflectance model, specularity

\section{INTRODUCTION}

Identifying planar surfaces in a single generic-scene image is important for image scene understanding, object recognition, and image forensics. With this capability, an image retrieval system can retrieve poster images regardless of their contents, a scene understanding system can better classify the geometry of a scene, and an image forensics system can detect rebroadcast attacks on a biometric identification system. Recently, a set of images of a tiger, which is believed to belong to an extinct species, has been in the spotlight in China [1]. Hypothesis has it that they are photos of a planar tiger cut-out. While checking homography is a way to verify this hypothesis on the set of images, our work forms the first step of an alternative method using scene specularity.

There are various monocular cues for estimating geometry from a scene: shading, specularity, texture orientation, shadows, silhouettes and so on. Shading cue is only reliable for uniform-color Lambertian surface, and texture orientation cue requires homogeneous texture on a surface. Both shadows and silhouettes can be easily confused with the non-uniform color on a surface, e.g., shadow within a poster. Specularity is the intense reflection of scene illumination from a surface and is a function of the surface orientation, when the viewing angle and the illumination direction are known. As specularity pertains to the illumination color, it can be more separable from a non-uniform color surface which is common in a generic scene. There are various techniques to decompose an image into a diffuse component and a specular component, even on a non-uniform color surface [2, 3, 4].

It was long known that specularity is uniform on a planar surface [5], under a distant point light source. However this clue becomes clouded in the generic scene when the illumination is complex and less intense, and the planar surfaces are of non-uniform color (e.g., a movie poster). In [6], Blake developed a stereo method to recover object geometry but the method requires two images of the object from different view points. In [7], specularity information is used to match an object in an image to a database of 3D object models. In [8], a study on how the specularity on a glossy surface provides constraints on the possible shape is conducted. However, all the above-mentioned methods are not sufficient for planar surface recognition in a generic scene.

In this work, we study a specific problem of planar surface recognition, that is distinguishing an image from its recaptured. A recaptured image is obtained by photographing the print-out of the image on a paper. It is a specific type of planar surface and, in this work, we assume the recaptured part occupies the entire image and hence no segmentation is needed. Our algorithm first decomposes an image into a diffuse component and a specular component using the technique in [3]. We find that the specular component on the recaptured surface contains some high-frequency spatial variations, which can be related to the micro-structure of the printing surface. We propose a cascaded dichromatic model to model this phenomena. We further define an objective measure to describe this distinct spatial feature. To eliminate the brightness difference due to the different linear scaling in the original and the recaptured, we compute the ratio of the specular image and the input image. Then, we are able to show that the distribution of the ratio image's gradient has a distinguishable form 
for the original and recaptured image.

The main contribution of our work is on showing the physical difference between an original image and its recaptured in their specular component. We relate this physical phenomena to the property of the printing surface and model it using a cascaded dichromatic model. Furthermore, this finding also suggests that the physical property of a surface can be examined through the specular component of the surface image. There are works on measuring the fractal dimension of a surface from its 2D intensity image [9], but analyzing the specular component of an image can be an alternative way to analyze a surface.

In Sec. 2, we introduce the dichromatic reflectance model and the technique to separate specular reflectance. In Sec. 3, we propose a cascaded dichromatic model for recaptured images. Experimental results are given in Sec. 4 on both the natural-scene original images and their recaptured counterparts. Finally, conclusions and future work are given in Sec. 5.

\section{BACKGROUND}

\subsection{Dichromatic Reflectance Model}

In natural scenes, a wide range of surface reflectance can be considered as a linear combination of diffuse and specular components. These surfaces includes plastics, cloth, human skin, leaves, and wood. In [10], a dichromatic model is proposed to model this type of surfaces, where the scene radiance of a surface point is given by

$$
I=c_{d} I_{d}+c_{s} I_{s}
$$

where $I\left(\left[I_{1}, I_{2}, I_{3}\right]\right)$ is the RGB color vector measured by a linear camera. $I_{d}$ and $I_{s}$ are respectively the diffuse and the specular color, while $c_{s}$ and $c_{d}$ represent the reflectance coefficients for the diffuse and the specular components. $c_{s}$ and $c_{d}$ depend on surface geometry and material properties. More details about the model can be found in [10].

\subsection{Separation of specular reflectance}

Based on a dichromatic model, the specular component can be separated by exploiting the differences between specular and diffuse components in color space. In our work, we use the method proposed by Tan et al. [3] which is demonstrated to be robust for images of complex scene and textured surfaces. Fig. 1 shows an example of diffuse-specular decomposition for a complex-scene image. Fig. 1 (b) and Fig. 1 (c) give the diffuse and specular components of the image in Fig. 1 (a).

There are a few assumptions for the method in [3]. First, the illumination is of uniform color. Second, there is no interreflection in the scene. However, as shown in [3], the method works even when the assumptions are not strictly satisfied.
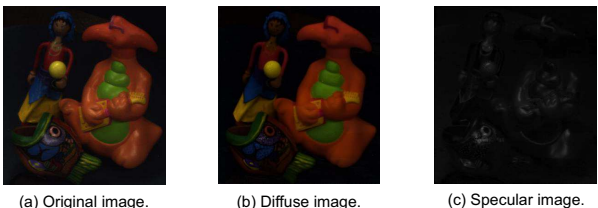

Fig. 1. Separation of specular reflectance on a complex scene.

\section{PROPOSED METHOD}

\subsection{Cascaded dichromatic model}

Our proposed method for detecting recaptured images is based on the physical modeling of a recapturing process. When a natural scene is first captured, the scene radiance measured by a camera consists of a diffuse component and a specular component from the objects in the scene (assuming a dichromatic model). For recapturing, the natural-scene image is printed on a paper or other printing medium, which is in turn recaptured by a camera under a probably different lighting condition. In this case, the recaptured scene radiance can be considered as a superposition of a diffuse component due to the non-uniform color of the original photo, and a specular component reflected from the photo paper surface. We model the recaptured image using a cascaded dichromatic model. Let $I_{1}=c_{d 1} I_{d 1}+c_{s 1} I_{s 1}$ (refer Eq. 1) be the original image. We consider $I_{1}$ form the diffuse component of the recaptured image. Hence, the cascaded model for a recaptured image $I_{2}$ can be expressed as:

$$
\begin{aligned}
I_{2} & =c_{d 2} I_{1}+c_{s 2} I_{s 2} \\
& =\left(c_{d 2} c_{d 1} I_{d 1}\right)+\left(c_{d 2} c_{s 1} I_{s 1}+c_{s 2} I_{s 2}\right)
\end{aligned}
$$

Note that the second part of Eq. 2, i.e., $\left(c_{d 2} c_{s 1} I_{s 1}+c_{s 2} I_{s 2}\right)$, corresponds to the specular component of a recaptured image and is a superposition of the original image specularity and the specularity of the photo paper. From the model, we expect that the specular component of a recaptured image will be distinguishably different from that of the original image. We will validate this effect in Sec. 4. In the model, we intentionally leave out the $2 \mathrm{D}$ perspective transformation on the recaptured image so that the effect of the superposed specular component can be more clearly illustrated.

\subsection{Specular Ratio}

The intensity level for an original image and its recaptured can be very different due to the probable dissimilar imaging condition. Then, comparing the value of the specular component may not be always meaningful, as it dependent on the intensity scaling. Therefore, we introduce a relative quantity called specular ratio to represent specularity in an image:

$$
r_{s}=I_{s} / I
$$

where $I_{s}$ and $I$ are the specular and input images. $r_{s}$ measures the proportion of specularity in the overall measured intensity, 


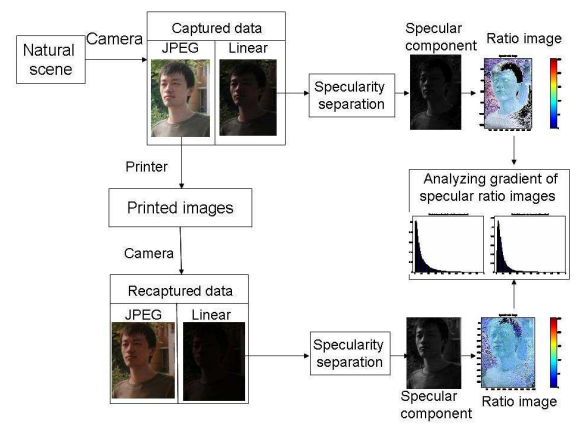

Fig. 2. The flow-chart of proposed method.

hence it is invariant to the intensity scaling.

\section{EXPERIMENTS}

\subsection{On Natural-scene Images}

In this section, we will validate the effect of superposed specular component for a recaptured image, as indicated by the cascaded dichromatic model in Sec. 3. The evaluation is performed using natural-scene original images obtained under various illumination environment, including indoor lighting and outdoor sunlight, whereas the recapturing is performed with a viewing direction orthogonal to the print-out of the original images under a white indoor area light of an medium intensity. The flow of our experiment is shown in Fig. 2, where given an image we will extract the specular component and compute the specular ratio. While the specular ratio of the original images and their recaptured are distinguishable, we further compute the distribution of the specular ratio's gradient and consider their shape as a distinguishing signature.

In our experiment, we use 20 pairs of original and recaptured images obtained under various lighting conditions. All the results are constant to support our hypothesis. Fig. 3 (a) lists four images with four typical illuminations which are outdoor ambient light, outdoor side light, indoor ambient light and outdoor frontal light. Fig. 4 (a) gives their recaptured images. Note that the recaptured images are visually similar to the original images. The specular component of the original images and the recaptured images are respectively given in Fig. 3 (b) and Fig. 4 (b). Fig. 3 (c) and Fig. 4 (c) give the specular ratio image. Fig. 3 (d) and Fig. 4 (d) give the histogram of the specular ratio's gradient.

Comparing Fig. 3 (d) and Fig. 4 (d), we can see that for the original natural-scene images, the histogram of the specular ratio's gradient generally follows a Laplacian-like distribution while that of the corresponding recaptured images follows a Rayleigh-like distribution. From Fig. 3 (c), the specular ratio of the original images consist of regions of relatively constant separated by conspicuous transitions. Such profile is similar to that of natural images and it is well-known that such profile will have a Laplacian-like distributions [11]. On the other hand, from Fig. 4 (c), we see the specular ratio of the recaptured images is similar to that of Fig. 3 (c) but it is superposed
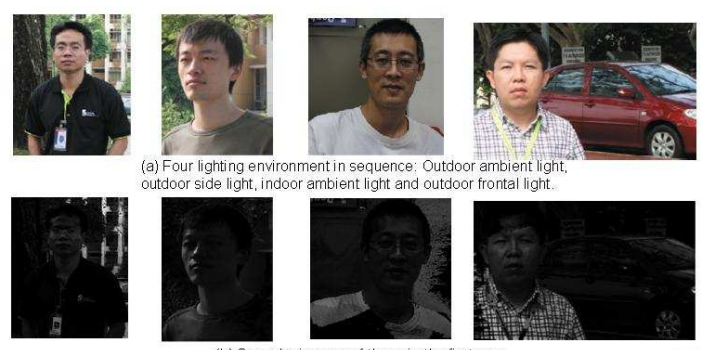

and outdoor frontal light.
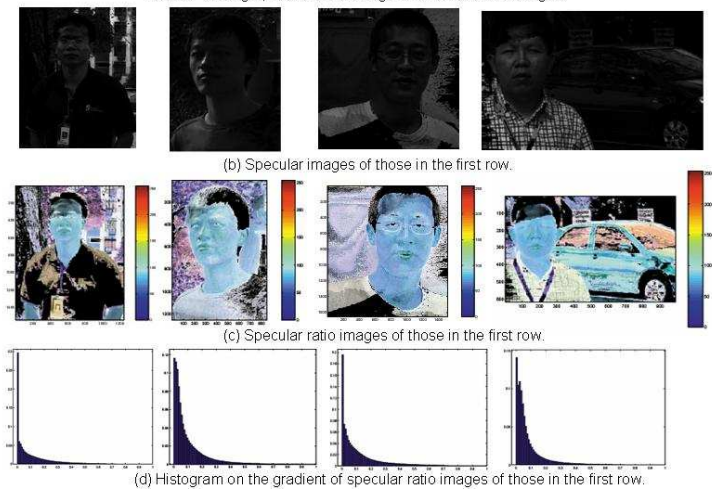

Fig. 3. Specularity analysis of images from natural scene.
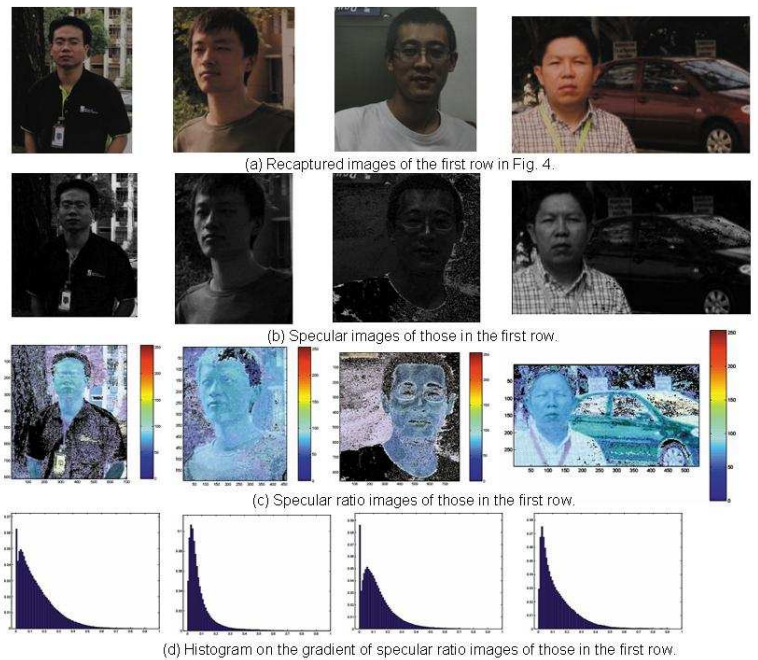

Fig. 4. Specularity analysis of images from recapturing.

with some high-frequency spatial variations. As a result, the specular ratio consists of less drastic transitions. Hence, it results in a departure from the Laplacian-like distribution for its gradient and arrives at a Rayleigh-like distribution which is reasonable.

\subsection{Relation to Paper Surface Property}

From the cascaded dichromatic model, the observed highfrequency spatial variations in the specular ratio of the recaptured image (Fig. 4 (c)) should be related to the printing paper surface property. To verify this, we perform an additional experiment on a plain printing paper. A printing paper is captured under the same lighting as described in Sec. 4.1. Fig. 5 (a) and Fig. 5 (b) shows the intensity and specular image of the paper. Note that the micro-texture manifested on specular component is spatially homogeneous, which is an important 
indication that the paper surface is planar. Fig. 5 (c) shows the specular ratio of the paper image and we can see that the micro-texture is similar to the high-frequency variation in Fig. 4 (c). Furthermore, Fig. 5 (d) shows the Rayleigh-like distribution of the specular ratio's gradient, which is similar to those in Fig. 4 (d). These observations on the paper image is a proof that the high-frequency variation in Fig. 4 (c) is related to the printing surface property and the Rayleigh-like distribution is a result of the high-frequency variation.
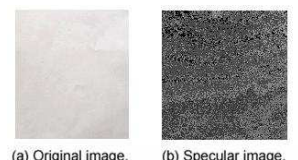

(b) Specular image.

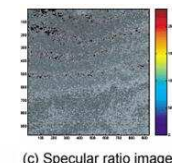

(c) Specular ratio image.

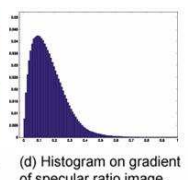

(d) Histogram on gradient
Fig. 5. Reflectance on a quasi-glossy paper.

\subsection{Experiment on the Controversial Tiger Image}

From Sec. 4.1, we observe that the shape of the specular ratio's gradient are different for original images and their recaptured, and it provides a signature for detecting recaptured images. We apply our method to tackle the controversial tiger images mentioned in Sec. 1, where one of the images is shown in Fig. 6 (d). A conjecture is that they are recaptured of a planar tiger cut-out, and hence we can apply our method to evaluate the conjecture. Fig. 6 (b) and Fig. 6 (e) respectively show the specular ratio of a real tiger image obtained from the Internet and that of the hypothesized planar tiger image. These images are originally non-linear, and radiometrically linearized using the camera response function estimated using method in [12], before diffuse-specular decomposition is performed. We can see that the suspicious tiger image is rejected to be a natural scene image given the Rayleigh-like distribution of its specular ratio's gradient. Whereas for the natural-scene tiger image, the specular ratio's gradient distribution is Laplacianlike, which is consistent with our hypothesis.

\section{CONCLUSIONS AND FUTURE WORK}

In this work, we discover a distinctive feature in the specular component of an image for recaptured photo detection. The distinctive feature is the high-frequency spatial variations in the specular component of a recaptured image, which is a result of the micro-structure on the printing paper. We explain this physical phenomena using a cascaded dichromatic model. We verify our findings using images of nature scene and their corresponding recaptured ones obtained under a generic illumination environment. The distinctive feature can be further identified from the form of the distribution of the specular ratio's gradient. For recaptured photos, the distribution is Rayleigh-like, while the distribution obtained from the original images follows a Laplacian-like distribution.

As the textureness in the specular component of a recaptured image is related to micro-structure of a planar surface,

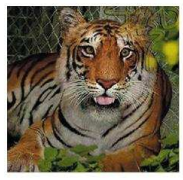

(a) A natural tiger image.

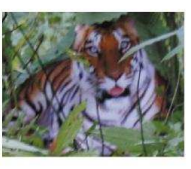

(d) A suspected tiger
image cut-out.
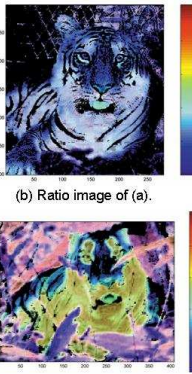

(e) Ratio image of (d)

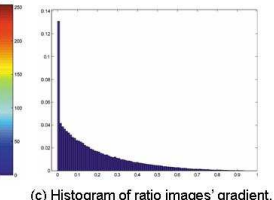

(c) Histogram of ratio images' gradient

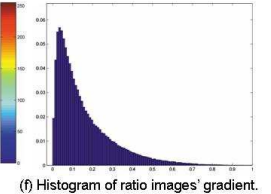

Fig. 6. Specularity analysis of a natural and suspicious tiger images.

in future work, we will model the pattern more formally so that it can account for a set of general surfaces with different coarseness. With this general model, we will be ready to apply our method to various types of surfaces. Furthermore, the specific superposed textureness in the specular component can be used for rendering the effect of a surface appearance. Finally with our finding, we can move forward to detect planar surfaces in generic scenes for scene understanding applications.

\section{REFERENCES}

[1] "Rare-tiger photo flap makes fur fly in china," Science, vol. 318, no. 5852, pp. 893, 2007.

[2] P. Tan, L. Quan, and S. Lin, "Separation of highlight reflections on textured surfaces," in IEEE CVPR, 2006, pp. 1855-1860.

[3] R.T. Tan and K. Ikeuchi, "Separating reflection components of textured surfaces using a single image," IEEE PAMI, vol. 27, no. 2, pp. 178-193, 2005.

[4] S.P. Mallick, T. Zickler, P.N. Belhumeur, and D.J. Kriegman, "Specularity removal in images and videos: A PDE approach," ECCV, vol. 1, pp. 550-563, 2006.

[5] G. Healey and T.O. Binford, "Local shape from specularity," Computer Vision, Graphics, and Image Processing, vol. 42, no. 1, pp. 62-86, 1988.

[6] A. Blake, Inferring Surface Shape by Specular Stereo., University of Edinburgh Department of Computer Science, 1984.

[7] M. Osadchy, D. Jacobs, and R. Ramamoorthi, "Using specularities for recognition," IEEE ICCV, pp. 1512-1519, 2003.

[8] R.W. Fleming, A. Torralba, and E.H. Adelson, "Specular reflections and the perception of shape," Journal of Vision, vol. 4, no. 9, pp. 798-820, 2004.

[9] P. Kube and A. Pentland, "On the imaging of fractal surfaces," IEEE PAMI, vol. 10, no. 5, pp. 704-707, 1988.

[10] S.A. Shafer, "Using color to separate reflection components," pp. 43-51, 1992.

[11] A. Srivastava, A.B. Lee, E.P. Simoncelli, and S.C. Zhu, "On Advances in Statistical Modeling of Natural Images," JMIV, vol. 18, no. 1, pp. 17-33, 2003.

[12] T.-T. Ng, S.-F. Chang, and M.-P. Tsui, "Using geometry invariants for camera response function estimation," in IEEE CVPR, June 2007. 